\title{
Mineral Accretion Factory ou la mer comme usine nouvelle
}

Mineral Accretion Factory or seabed as new factory

David Enon

\section{(2) OpenEdition}

1 Journals

Édition électronique

URL : https://journals.openedition.org/tc/8491

DOI : $10.4000 /$ tc. 8491

ISSN : 1952-420X

Éditeur

Éditions de l'EHESS

\section{Édition imprimée}

Date de publication : 6 juin 2017

Pagination : 240-257

ISBN : 978-2-7132-2707-3

ISSN : 0248-6016

\section{Référence électronique}

David Enon, «Mineral Accretion Factory ou la mer comme usine nouvelle », Techniques \& Culture [En ligne], 67 | 2017, mis en ligne le 06 juin 2019, consulté le 29 septembre 2022. URL : http://

journals.openedition.org/tc/8491; DOI : https://doi.org/10.4000/tc.8491 


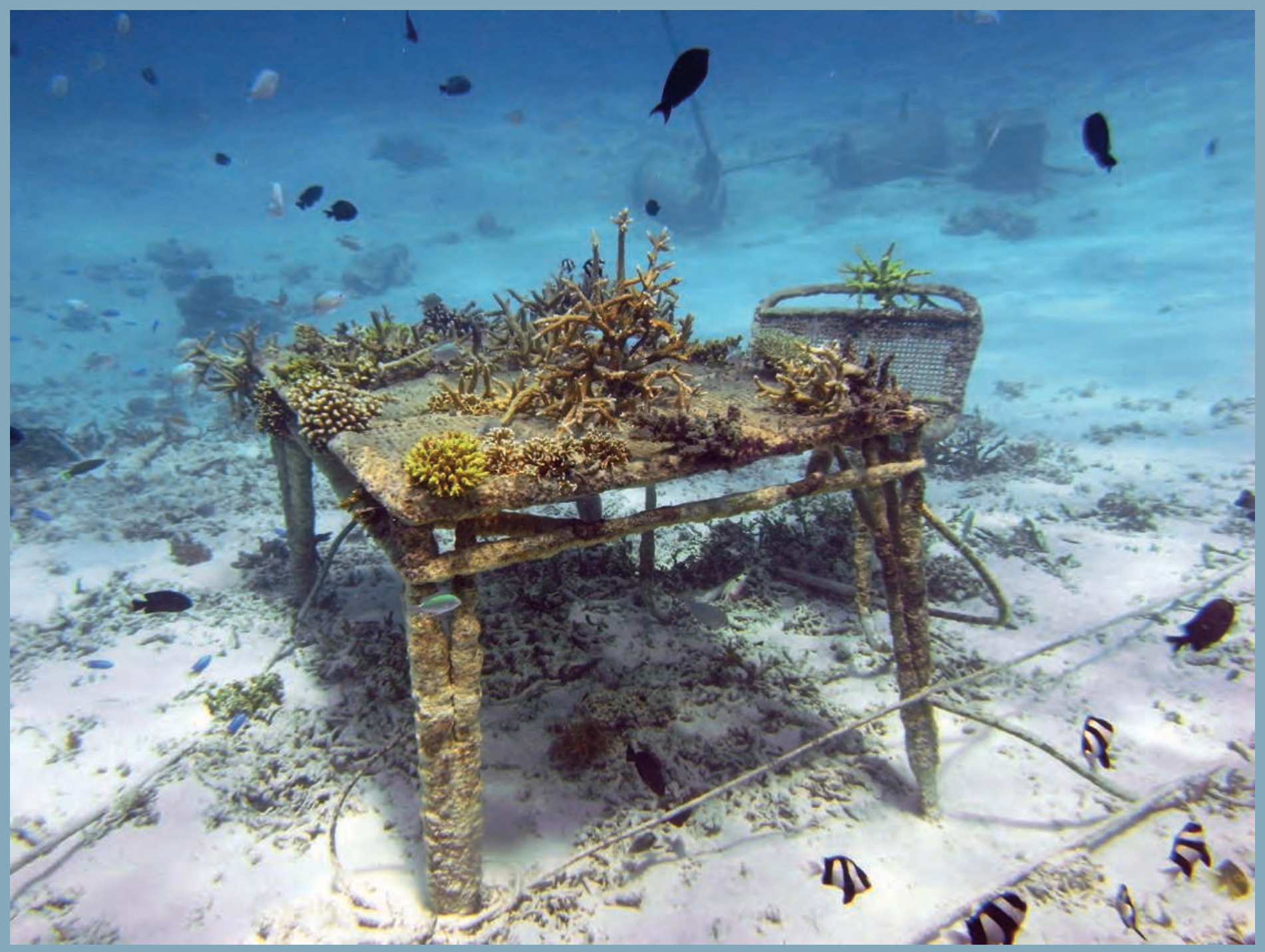




\section{Mineral Accretion Factory ou la mer comme usine nouvelle}

Quel progrès voit-on au gain de quelques centièmes de secondes dans la fabrication d'une pièce plastique complexe de produit de grande consommation ? Les logiques de production à l'œuvre ont trouvé leurs limites. Le rôle du designer c'est aussi de travailler à éviter l'ajout d'un objet supplémentaire au monde, prôner de manière argumentée de ne pas produire ${ }^{1}$ ou préconiser de produire autrement selon des critères d'efficacité qui dépassent les logiques de profit à court terme.

Tout un pan de la pratique du design se détache de l'industrie, ce qui dans le même temps ravive son sens politique. Le design est un investissement pratique de notre environnement : cette pratique n'est pas la simple « phase » préliminaire d'un processus, mais elle se distribue à travers tous ceux et celles qui interagissent avec les objets. Mais comment participer de l'hétérogénéisation et de la diversification des modes de production dont nous avons besoin ? S'interrogeant sur les formes à donner à notre environnement matériel et par extension à ses façons de faire, le designer se doit d'élargir son champ d'appréhension en expérimentant toutes sortes de dispositifs de fabrication, des plus alternatifs et singuliers aux plus communs. De l'autoproduction (établissement d'un design diffus porté par tous) à tous les modes de productions qui se déploient par le bas grâce à la mise en réseau de savoirs et de savoir-faire, et par le haut soutenu par la quantité de production.

L'économie de gestes et de moyens, tout comme les politiques de recyclage, de réemploi à l'œuvre, si elles se trouvent être une nécessité, ne suffisent plus. Une piste à envisager serait de travailler l'inscription des projets dans une continuité, dans le temps, pour qu'ils soient vécus in situ. Reprendre le temps de prendre le temps, en considérant le développement (l'acte de développer) du projet, de sa conception à sa fabrication (sa mise en forme) comme étant tout aussi important, si ce n'est plus, que le résultat (un objet fabriqué et décontextualisé). Ce temps, que l'on s'est évertué à raccourcir, à faire disparaître, à décimer, ce temps combiné de 
la pensée et du faire peut être investi collectivement et constituer une plus-value sociale et, de fait, économique. Le développement est aussi le résultat du projet. Il faut envisager de mesurer l'efficacité d'un projet selon d'autres critères que l'optimisation de sa production matérielle.

Il s'agit non seulement d'une logique d'appropriation de la technologie, une réduction à sa plus simple expression qu'on appelle low tech (qui ne signifie pas un reniement du progrès technologique mais plutôt un usage à bon escient), mais accompagnée d'une dimension temporelle propre qui permette d'être davantage en accord avec un rythme de production biologique des formes et de la matière que l'on pourrait nommer slow tech.

Considérons l'autogenèse ${ }^{2}$. Il s'agit, à l'origine, d'une « vieille doctrine » selon laquelle des formes de vies peuvent apparaître de novo, sans aucune sorte d'origine. C'est une notion toujours en usage en biologie. Dans ce contexte il s'agit d'une genèse qui se produit sous la propre impulsion de l'organisme, sans influences extérieures. Abordée sous l'angle de la production, l'autogenèse est une utopie. Celle d'une matière qui s'autogénère, s'auto-organise. Le rêve d'un objet qui s'autoproduirait totalement. Une autoproduction où l'intervention de l'homme serait réduite a minima. Donner l'impulsion la plus petite possible et la matière s'agencerait d'ellemême tranquillement, paisiblement. Ce processus de composition slow tech qui va au-delà du low tech, tend vers une utopie du no tech.

Si ces enjeux semblent n'être que pure utopie, je travaille, de manière bien plus modeste et avec des capacités opératoires singulières qui ont le mérite de s'engager sur cette voie. Comme un début, un chemin de traverse, qui, dans la limite de son potentiel, de ses spécificités matérielles, à contre-courant de la plupart des logiques industrielles à l'œuvre, je développe depuis près d'une demi-douzaine d'années un projet de production d'objets par accrétion minérale en détournant de son usage principal un système de récifs artificiels appelé Biorock.

\section{Du récif artificiel comme usine de production nouvelle}

\section{L'inquiétante disparition des coraux}

Les coraux, petits animaux de la famille des cnidaires, vivent en colonies, majoritairement dans les mers chaudes. Ils ont comme propriété de fabriquer leur exosquelette, calcaire et dur, formant ainsi des récifs.

Les récifs coralliens occupent à peine 0,1\% de la surface des océans (soit 1,2\% des plateaux continentaux), et abritent une biodiversité exceptionnelle aux fonctions écologiques essentielles. Ils offrent un panorama sans pareil de la vie sous-marine : coraux, poissons, raies, tortues, requins. Plus de $25 \%$ de la biodiversité marine mondiale y évolue ; de très nombreuses espèces en dépendent.

Les récifs coralliens jouent un rôle très important dans l'absorption du $\mathrm{CO}_{2}$. S'il est d'usage de dire que la forêt amazonienne est le poumon de la terre, c'est l'océan qui est le plus grand puits de carbone. L'océan absorbe le carbone de l'atmosphère et contribue à la diminution du taux de $\mathrm{CO}_{2}$; 
près de $50 \%$ de l'absorption de $\mathrm{CO}_{2}$ est assimilé via le plancton, les poissons et les coraux.

Les récifs coralliens forment généralement des lagons qui jouent le rôle de zones tampons entre le large et la côte. Ils protègent de l'érosion des vagues ou même de celle provoquée par les ouragans. Toute une partie de la population littorale et insulaire dépend directement des récifs. L'essentiel de la production alimentaire et des revenus issus de la pêche et du tourisme balnéaire en est issu.

Les coraux, espèce protégée très fragile, sont extrêmement sensibles au réchauffement climatique. Si la température de l'eau augmente de $1{ }^{\circ} \mathrm{C}$, le corail blanchit, si elle augmente de $2{ }^{\circ} \mathrm{C}$, il meurt ${ }^{3}$. Le corail est aussi sensible à l'acidification des eaux de l'océan, phénomène en corrélation avec la montée des températures. En 1998, l'augmentation d'amplitude d'El Niño ${ }^{4}$ également liée au changement climatique a eu des conséquences désastreuses. Au-delà des feux de forêt, pluies diluviennes, inondations, tornades, tempêtes de neige, etc., des centaines de kilomètres de récifs coralliens ont péri. En mars 2016, 93 \% de la grande barrière de corail ${ }^{5}$ était touchée par le blanchissement. Si la sensibilité des coraux au réchauffement du climat est vérifiée, l'homme reste néanmoins l'un

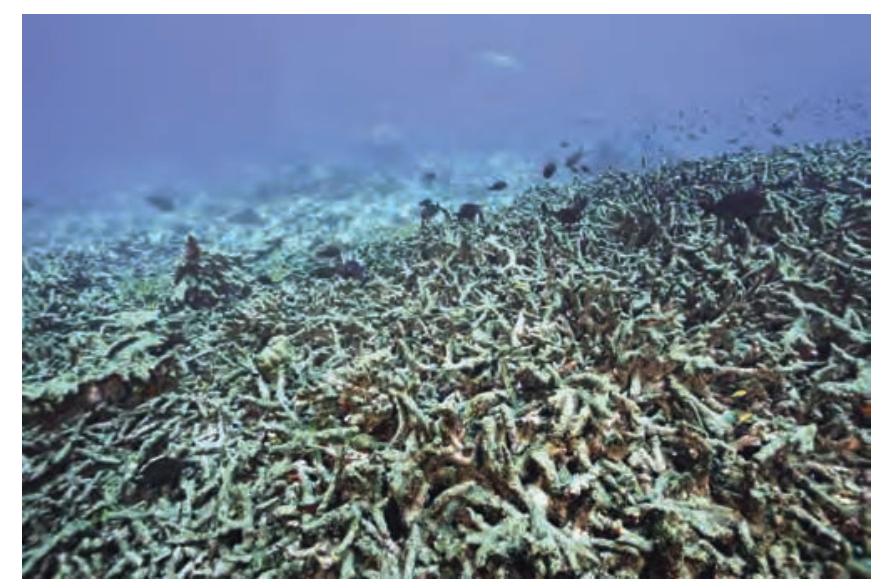

1. Une grande partie des récifs coralliens sont aujourd'hui décimés à cause du réchauffement climatique et de l'acidification des eaux marines.

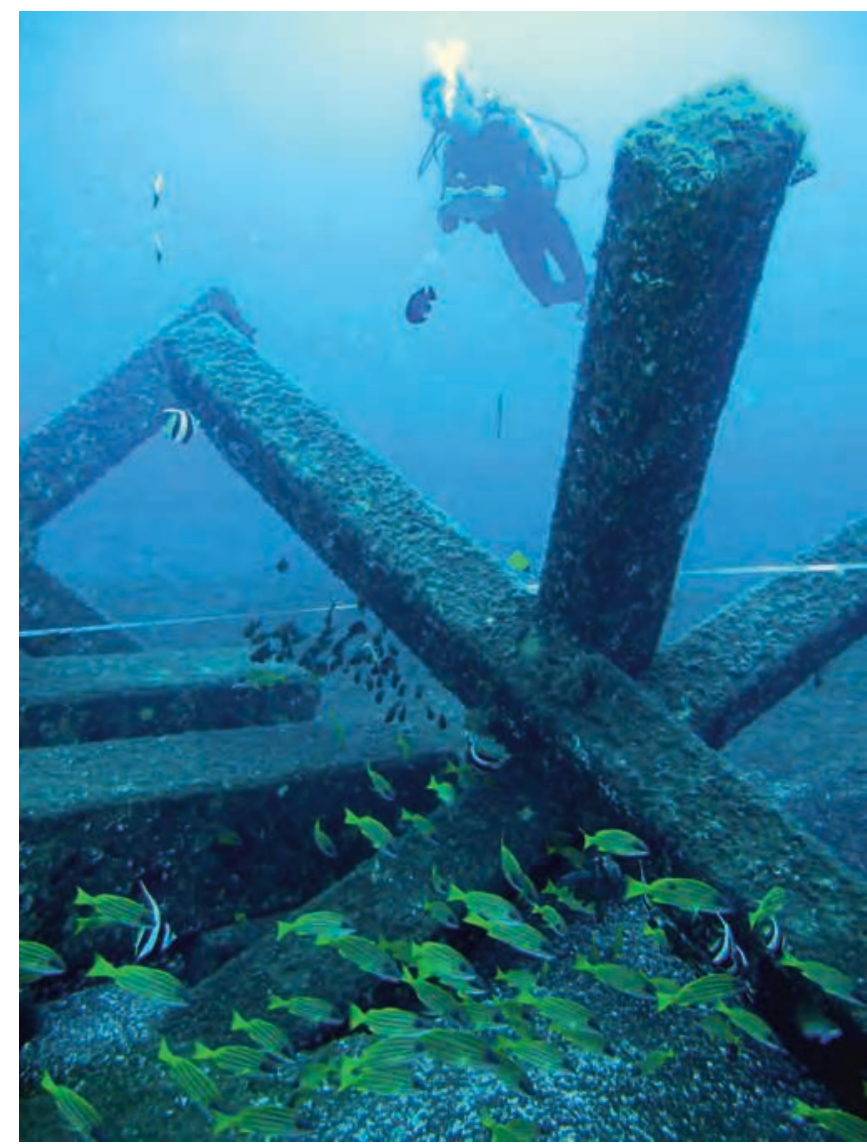

2. Les récifs artificiels produits en France sont généralement en béton armé. Souvent des modules existants comme des buses sont enchaînés en grappes. Parfois des modules sont créés à cet effet. A La Réunion, des poteaux EDF en ciment recyclés ont été assemblés. 
des plus grands destructeurs de récifs. Certains modes de pêche au chalut, au cyanure ou à la dynamite par exemple déciment les récifs. Les barrières sont aussi détruites pour ouvrir des accès en bateau à des complexes hôteliers en bord de mer.

\section{Les récifs artificiels}

Les récifs artificiels ont été conçus pour réparer les dommages créés par l'Homme. Selon la définition de l'Ifremer ${ }^{6}$ donnée en 2000, ils désignent « des structures immergées volontairement dans le but de créer, protéger ou restaurer un écosystème riche et diversifié. Ces structures peuvent induire chez les animaux des réponses d'attraction, de concentration, de protection et, dans certains cas, une augmentation de la biomasse de certaines espèces $»$.

Le principe est simple : il suffit d'observer comment la faune et la flore investissent une épave. Les récifs artificiels constituent un moyen de lutter contre la désertification de zones des fonds marins trop sollicitées par l'homme ou abîmées par la pollution.

Ils permettent également de préserver le littoral, de limiter l'érosion des côtes et de minimiser les migrations de sable autant que de réparer les dommages causés par des aménagements peu scrupuleux. Il existe différentes formes de récifs artificiels : épaves (bateaux, bus, tanks et tout autre véhicule encombrant), agencements de pneus usagés, etc. Sous prétexte de préserver les fonds marins et le littoral, des abus ont été commis. Certaines interventions allaient jusqu'à ressembler à des décharges sous-marines. C'est a posteriori que la toxicité de certaines opérations s'est révélée. De nouvelles préconisations ont été faites : les objets servant de récifs artificiels doivent désormais être conçus dans des matériaux inertes.

En France, les systèmes de récifs artificiels développés sont pour la plupart composés de masses de béton, plus ou moins organisées et structurées. Ce sont souvent des principes assez lourds à mettre œuvre et en place. La mise à l'eau de pièces de béton nécessite d'importants moyens de manutention. Et si le béton est un matériau inerte, il n'en reste pas moins une matière étrangère au milieu marin, dont les quantités immergées sont ici colossales.

\section{Les récifs artificiels Biorocks}

Les récifs artificiels Biorocks naissent de la rencontre entre Thomas J. Goreau et Wolf Hilbertz.

Thomas J. Goreau est docteur, chercheur en biogéochimie ${ }^{7}$. Ses recherches traitent à la fois de l'impact du changement climatique et de la pollution sur les coraux mais aussi des nouvelles maladies qui se développent dans les récifs des Caraïbes, de l'océan Indien et du Pacifique. Ses travaux récents portent sur la restauration des récifs coralliens, la mariculture et la protection du littoral ${ }^{8}$.

Wolf Hilbertz ${ }^{9}$ (1938-2007), architecte et biologiste, a développé de nombreux projets d'architecture prospective comme Autopia Ampere [Auto + Utopie + Ampère], qui s'inscrit dans un 
travail de recherche autour des concepts de Cybertecture et de Responsive Environnement qu'il a développé au sein du Responsive Environnement Laboratory ainsi qu’au Symbiotic Process Laboratory, laboratoires de recherches qu'il a successivement fondés. Même si ces projets architecturaux n'ont jamais été réalisés, ils s’appuient sur un principe constructif concret : l'accrétion minérale.

Laccrétion minérale est une réaction d'oxydoréduction en milieu marin. Elle consiste en un échange d'électrons entre deux électrodes (généralement métalliques) dans une solution aqueuse. Elles permettent par exemple de dorer des boutons métalliques ou des porte-clés.

Un courant de faible tension ( 2 à 6 volts) circule entre deux électrodes : la cathode et l'anode. La cathode est composée d'une structure en acier, généralement du fer à béton, matériau à bas coût utilisé partout dans le monde. L'anode, quant à elle, est constituée d'une petite quantité de grillage de titane, matériau technique plus difficile à trouver dans le commerce. Une fois les électrodes immergées et le courant activé, la réaction chimique démarre. Le carbonate de calcium $\left(\mathrm{CaCO}_{3}\right)$ présent dans la mer, ce même calcaire avec lequel le corail fabrique son exosquelette, se dépose sur la cathode. Ce dépôt, obtenu par la réaction électrochimique, protège immédiatement le fer à béton de la corrosion. Ensuite, lentement, la matière s'agglomère sur la structure et durcit. En trois ans, avec une base en fer à béton d'environ 10 millimètres de diamètre, on obtient un matériau composite de 30 à 40 millimètres de diamètre. La structure est alors stabilisée, il n'est plus nécessaire de l'alimenter en courant.

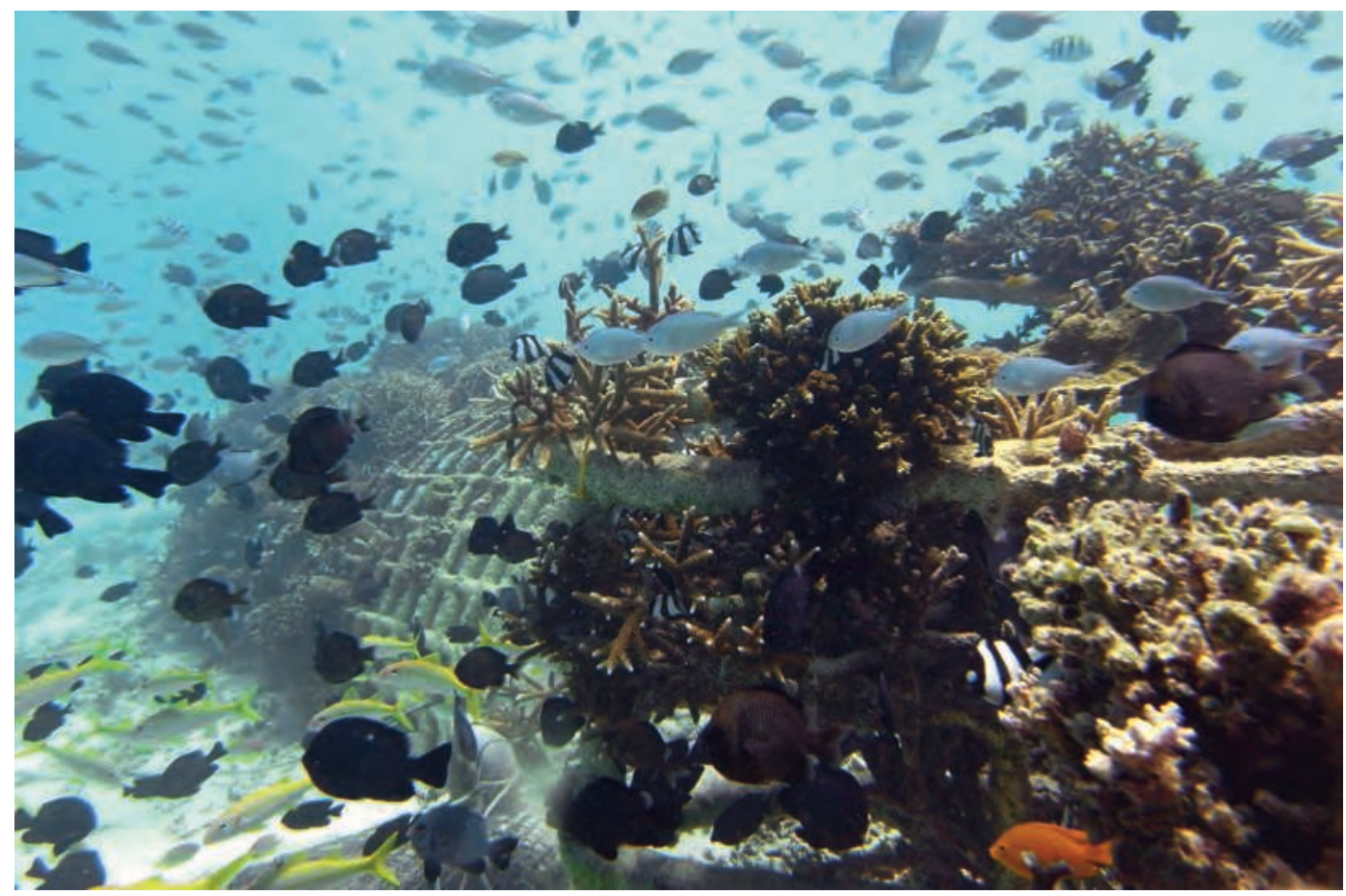

3. Les récifs artificiels Biorock sont des structures légères réalisées en fer à béton, un matériau peu coûteux et largement disponible partout dans le monde. Sa mise en œuvre est facile. Ce principe évite de déverser des tonnes de béton à la mer. 
Thomas J. Goreau comprend que l'on peut construire des récifs artificiels grâce à ce dispositif. Structures légères, matériaux peu coûteux et largement disponibles, mise en ouvre facile : il n'est plus nécessaire de jeter à la mer des tonnes de béton.

Le biologiste et l'architecte, définissent les principes du système de récifs artificiels Biorock. Ils peaufinent le système d'accrétion minérale au service d'un nouveau type de récifs artificiels. À défaut de construire des habitats sous-marins pour l'Homme, ils définissent les principes de cités pour poissons, crustacés, coraux, anémones, et tout autre élément de la faune et de la flore subaquatique.

Les récifs Biorock peuvent être construits sur une plage investie comme un atelier de production temporaire. Il suffit d'un accès au courant électrique pour brancher un poste à souder à l'arc. Une pince coupe boulon dite «monseigneur » ou une meuleuse pour découper les fers et des griffes pour les tordre et les façonner suffisent à construire les structures de base du récif Biorock.

Ces structures ne demandent pas une précision particulière, et sont rapidement fabriquées. Elles sont légères et faciles à manipuler par deux ou trois personnes. Elles peuvent aisément être hissées sur de modestes embarcations pour être acheminées sur le site d'implantation.

Les structures sont mises à l'eau avec des plongeurs ou un système de bouées. Les plongeurs vont ensuite aux abords des récifs naturels récolter des coraux détachés des récifs mais encore en vie. Ils les rapportent et les attachent avec du fil de fer sur les structures fraîchement immergées. On parle de bouturage. La cathode est prête.

Lanode, fabriquée avec quelques dizaines de centimètres carrés de grillage de titane roulé, est placée à proximité. Les deux électrodes sont ensuite raccordées à une source de courant électrique basse tension. Le courant peut être tiré d'une échoppe de bord de mer, raccordé à l'éclairage public ou issu d'un groupe électrogène.

Les récifs sont rarement éloignés de plus de quelques dizaines de mètre de la côte et immergés par 3 à 10 mètres de fond. Dans le cas d'implantation dans une zone dépourvue de réseau électrique, on utilise des bouées munies de panneaux solaires ou d'éoliennes.

Les électrodes connectées, la réaction s'enclenche. Des bulles d'hydrogène se forment autour de la structure. Elles témoignent du bon fonctionnement du dispositif. Au bout de quelques heures, la structure en fer à béton est recouverte d'une pellicule de calcaire blanchâtre. Les poissons investissent instantanément le récif. En quelques mois la structure est généreusement enrobée mais la matière ajoutée reste fragile. Les coraux s'installent et se développent. Il faut attendre plusieurs mois pour que le dépôt calcaire durcisse et devienne un matériau résistant.

Au départ souple et fragile, la structure devient plus résistante que le béton armé.

Le faible courant électrique qui parcourt la structure en fer à béton concentre le carbonate de calcium : il permet de renforcer la structure du récif mais il est aussi nécessaire à la construction de l'exosquelette du corail et stimule sa croissance. La croissance du corail s'y réalise cinq fois plus vite. Les observations montrent que de nombreuses espèces de la flore marine telles que les posidonies ${ }^{10}$, s'y développent aussi plus rapidement. 


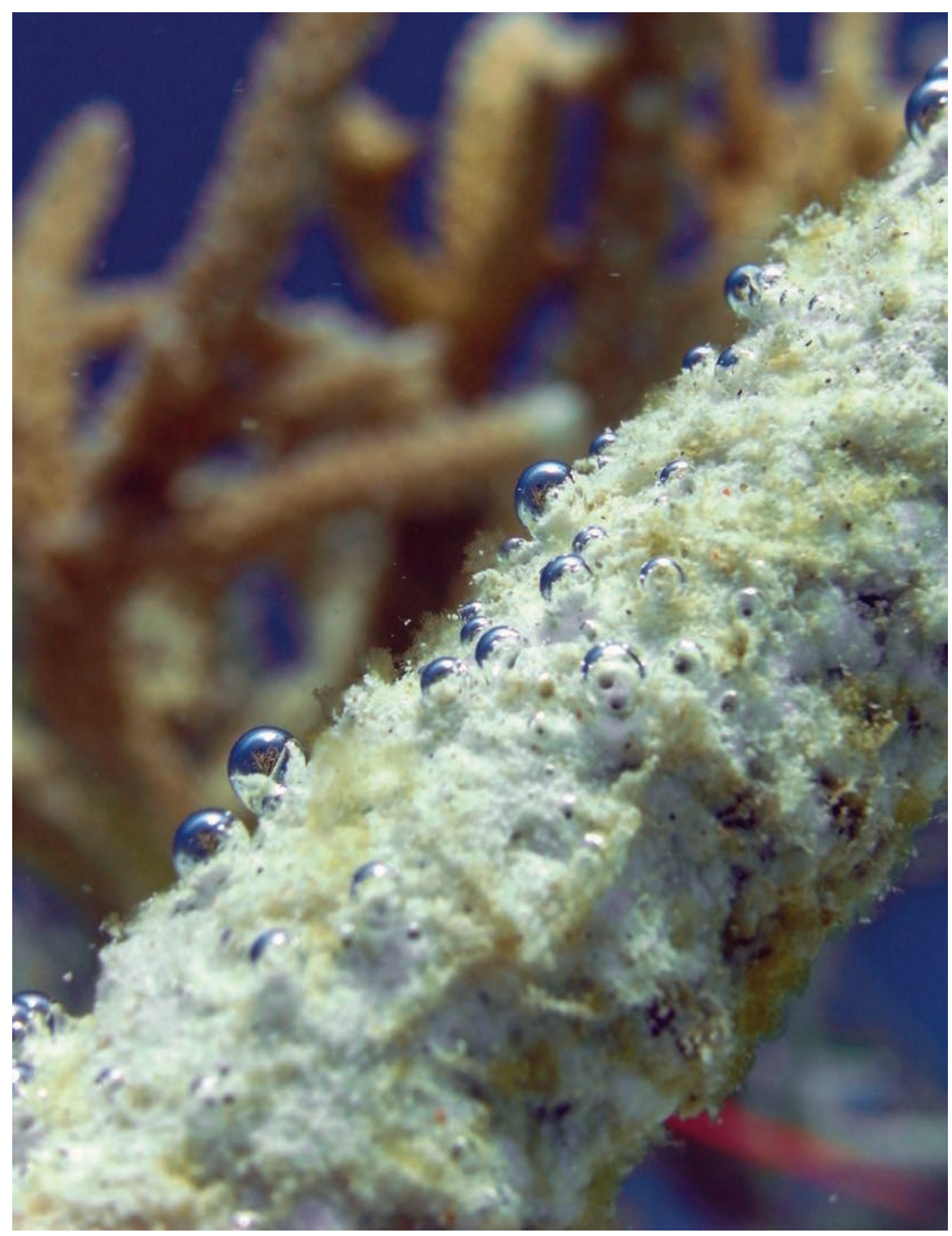

4. De petites bulles d'hydrogène se forment autour de la structure. Elles témoignent du bon fonctionnement du dispositif d'accrétion minérale. 


\section{De Mineral Accretion Furnitureà Mineral Accretion Factory}

La construction d'objets par accrétion minérale ouvre un champ de possibles. En dessinant des éléments en fer à béton et en grillage tels de fragiles squelettes, des mobiliers à venir peuvent prendre corps. Pour ce faire, il suffit de considérer l'échelle et l'usage, anticiper l'agglomération du carbonate de calcium et jouer de la dimension organique et aléatoire du procédé. S'inspirer du système Biorock pour produire du mobilier demande en effet, de penser l'intégralité du processus : du dessin de l'objet jusqu'à son utilisation après la sortie de l'eau. Tout comme les récifs, on peut imaginer immerger des squelettes d'objets, dans un endroit où la mise en place est bénéfique, de les brancher et d'y bouturer du corail. Deux à trois ans plus tard, on obtient du mobilier résistant aux contraintes d'usage. On peut alors sortir le mobilier de l'eau. Afin d'éliminer les micro-organismes, figer l'objet et anéantir l'odeur marine, j'imagine une exposition à haute température (soleil, étuve...). Un minimum de finitions serait sans doute nécessaire pour rectifier les surfaces utiles, aplanir les assises de chaises ou les plateaux de table par exemple. Un recouvrement de ces surfaces par un produit hydrofuge comme il est d'usage pour les marbres, granits et autres matériaux minéraux viendrait clore le traitement.

Il me semble essentiel d'envisager cette production selon un cycle rigoureux afin de perturber le moins possible l'écosystème. Avant de retirer un objet de l'eau, il faut réimplanter les coraux sur une nouvelle structure en fer à béton vierge. Le mode de gestion à mettre en place est à comparer à celui d'une forêt. Lorsqu'une forêt est bien gérée, le prélèvement de bois s'avère négligeable pour l'écosystème.

C'est sur l'île de Gili Trawangan dans le cadre du $8^{e}$ Indonesian Biorock reef restoration training workshop ${ }^{11}$ que je fabrique et implante le premier prototype de table et de chaise en accrétion minérale.

Située en Indonésie, l'île de Gili Trawangan a un périmètre de sept kilomètres. Ses habitants ${ }^{12}$ vivent de la pêche et du tourisme organisé autour de la plongée. La zone dite du « triangle d'or » dans laquelle se trouve l'île est un « spot» de renommée internationale. On y mesure l'une des plus grandes biodiversités sous-marines planétaires. Pour la préserver, un dialogue s'est engagé entre les plongeurs et les pêcheurs. À chaque plongée effectuée à Gili, cinq dollars sont prélevés et redistribués à l'association des pêcheurs. En échange, ces derniers se doivent de respecter un mode de pêche traditionnel. Dans la continuité, de nombreuses initiatives écologiques ont été mises en place, par exemple l'incitation politique à l'abandon de l'usage des sacs plastiques, ou l'aménagement des fosses septiques.

Que la question écologique soit ainsi posée, traitée et partagée sous la forme d'un investissement rentable et concerté, est, dans des territoires pauvres, un fait rare.

Le contexte s'est avéré propice au développement d'un tel projet. Cette expérience a permis de conforter les hypothèses du projet et développer les connaissances nécessaires à la maîtrise du processus de fabrication de récifs artificiels Biorock in situ. À ce jour, table et chaise sont 
encore immergées. Elles pourraient être sorties de l'eau. Mais elles restent, pour l'instant, les témoins de la première étape du projet.

C'est sur l'île de La Réunion que s'ouvrent de nouvelles perspectives. Un projet de recherche s'engage avec l'école supérieure d'art de La Réunion. L'objectif est d'engager le principe de Mineral Accretion Furniture vers la mise en place d'une unité de production sous-marine opérationnelle : Mineral Accretion Factory.

La première tentative de production tangible d'objets se met en place dans les bassins de Kélonia à Saint-Leu. Kélonia est à la fois un aquarium, un musée, et un centre de recherche, d'intervention et de soins consacré aux tortues marines. C'est un lieu privilégié d'expérimentation. Ses bassins en bord de mer, dont l'eau est renouvelée continuellement par des pompes, proposent des conditions idéales, dignes d'un laboratoire prévu à cet effet, pour lancer les premières productions.

Un deuxième temps dédié au lancement de la production de mobilier pour les espaces extérieurs de Kélonia est prévu en 2017.

\footnotetext{
Maquette échelle $1 / 1$ du dispositif M.A.F. pour une table avant immersion

(squelette de table en fer à béton + câble et électrode + plateforme solaire flottante) Vue de l'exposition Analog à l'esba TALM, site d'Angers
}

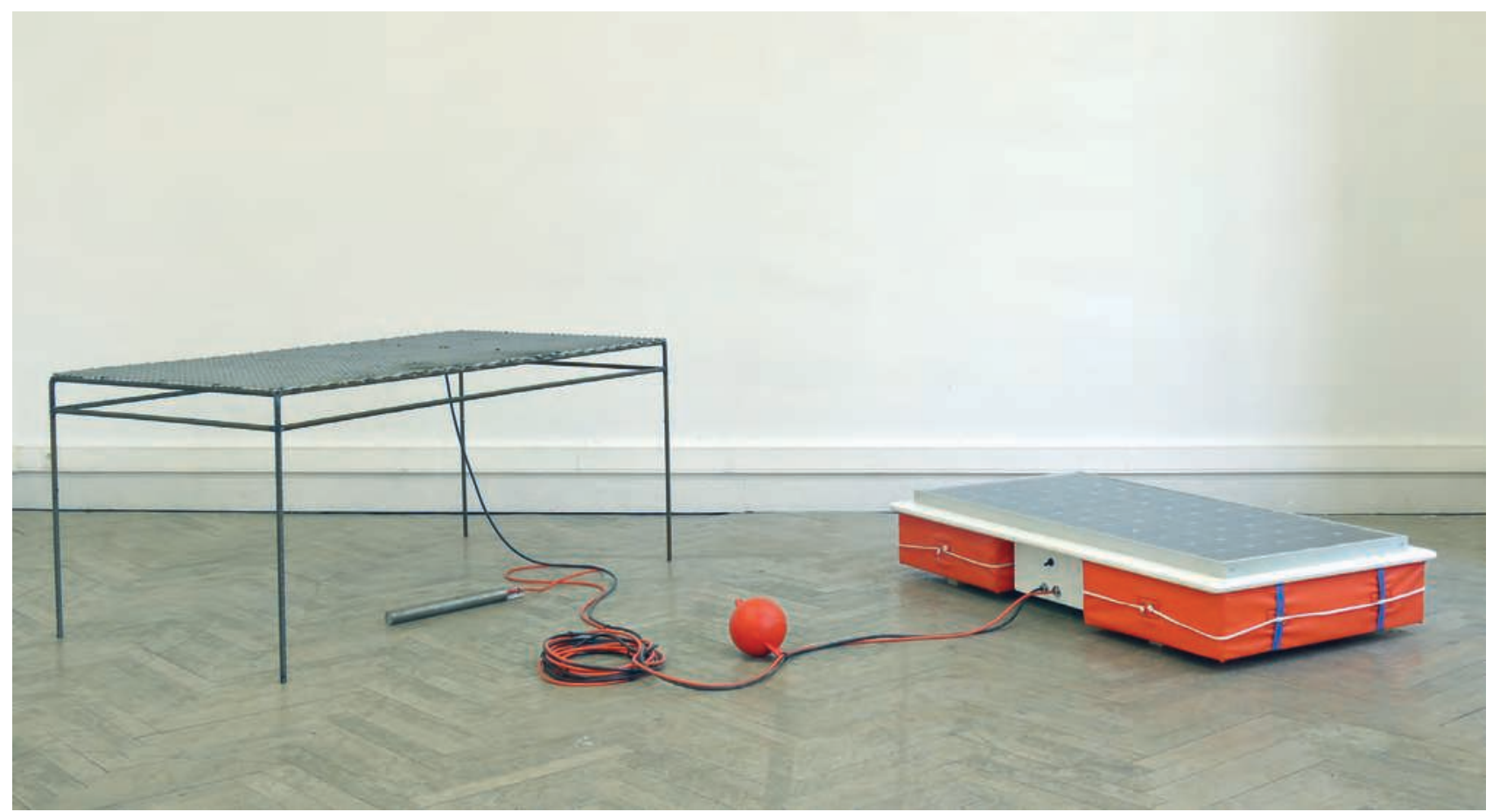


Mineral Acretion Factory est une tentative de mise ne place d'un système de production alternatif d'objets matériels. La typologie des objets qu'il est possible de produire est singulière et ses capacités restent restreintes. Elle vaut surtout dans sa participation à la diversification des modes de production des formes de notre environnement. Elle pose singulièrement la question d'une production in situ dont l'intérêt réside particulièrement dans son impact écologique positif. C'est un système low tech et slow tech qui respecte les rythmes biologiques et les capacités de production de la Terre. Si l'usine devient la mer, aucune implication locale n'est à déplorer (bâtiment à construire, parking, voie d'accès, poubelles, chauffage, climatisation...). L'emprise au sol est ici vertueuse puisqu'elle participe de la reconstruction du récif corallien et accompagne le développement de la faune et de la flore marine, sans l'implication d'une main-d'œuvre importante ni les inconvénients d'une forte activité humaine.

C'est un mode de production d'objet qui peut s'adapter à des échelles variées. Il peut servir à l'aménagement du littoral en produisant des éléments de mobilier urbain ou des éléments plus lourds tel que des digues. Il peut aussi participer au développement d'un artisanat contemporain tourné vers le futur plutôt que vers une folklorisation maladroite et mercantile de traditions éculées.

Pour le designer, ce type de dispositif renverse la question du dessin qui ne peut plus prévaloir sur l'utilité. La structure découle d'un dessin minimum et primitif, simple squelette d'objet dont la forme finale dépend plus de l'aléatoire du processus que du dessin initial.

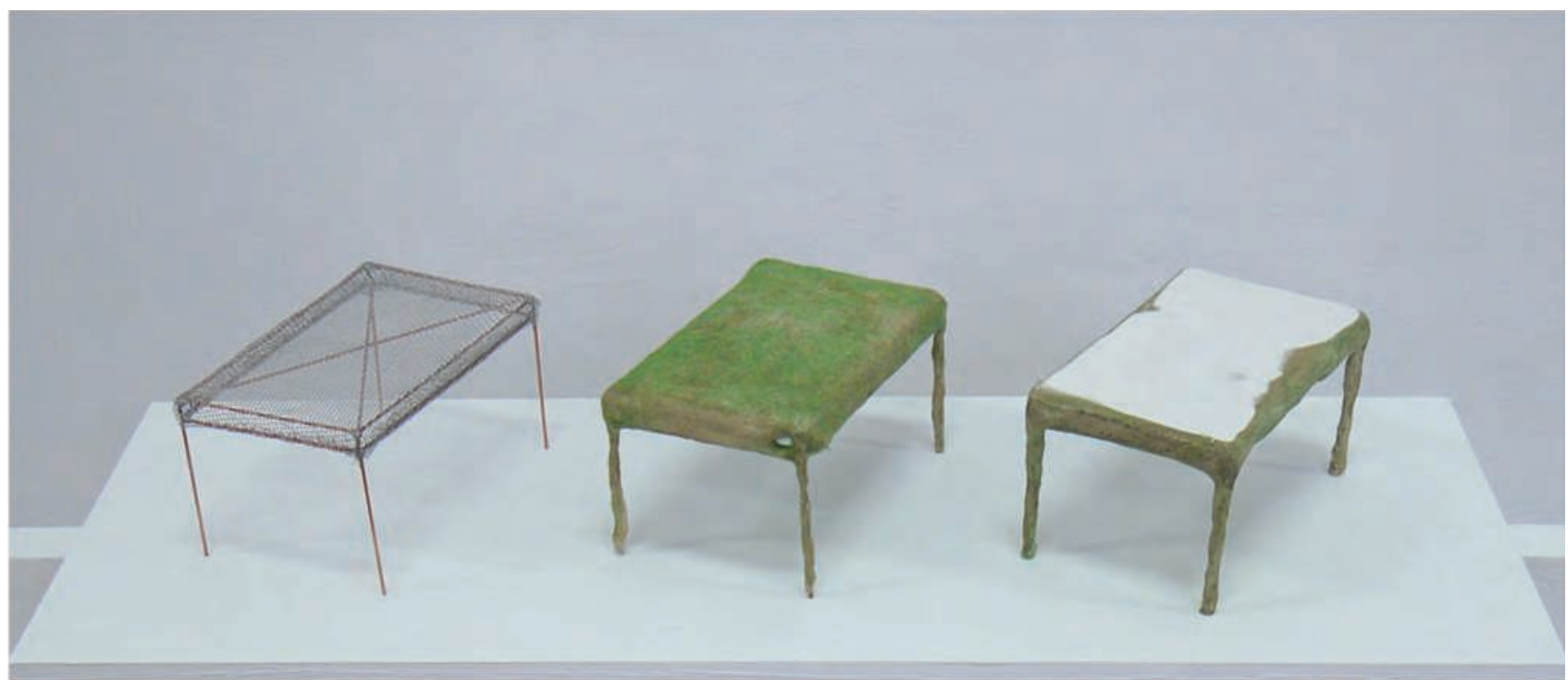



(1) (1) 垈,

\section{MINERAL ACCRETION FURNITURE}

The Mineral Accretion Process was invented by architect and biologist Wolf Hilbertz to grow construction materials from ocean'minerals.

Wolf Hilbertz and Dr. Thomas J. Goreau - President of the Global Coral Reef Alliance - both develope the process $\left(\right.$ Biorock $^{\ominus}$ ) to create artificial reef to help the coral reef restoration (almost $64 \%$ of the corals died during the 20 past years), fisheries restoration, shore protection and mariculture.

The process is based on structures easily built with locally available conductive material (usually rebar) which is submerged.

A low voltage electric power is applied between the conductive structure (cathode negative) and a pièce of titanium (anode positive). Minerals crystallize from seawater onto structures. The structure is then, auto-protected and the calcium carbonate white limestone $(\mathrm{CaCO} 3)$ grow quickly on it.

The purpose of Mineral Accretion Furniture is to use this low tech and slow process to create furniture and domestics objetcs according to a biological rhythm.

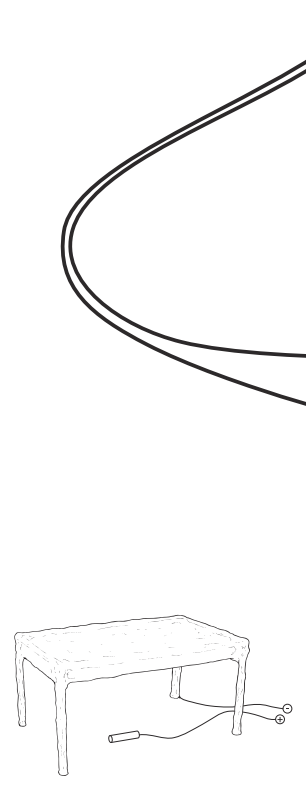

PROCESS

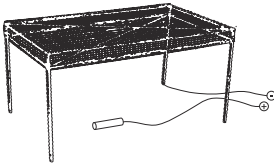

1_Submerging the structure
2_Leaving the structure 2 or 3 years
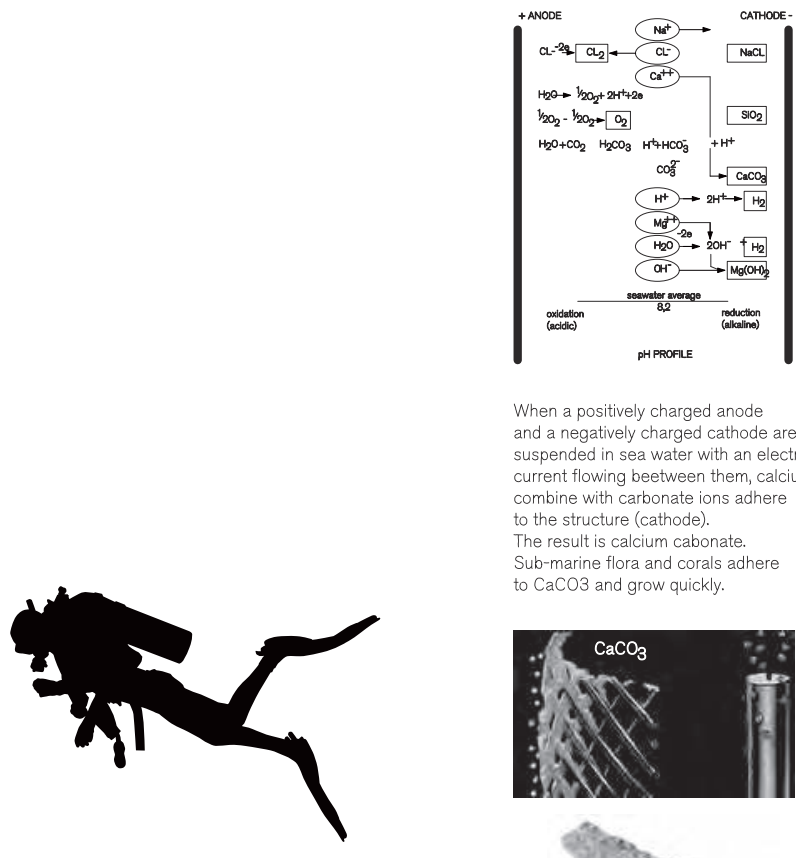

When a positively charged anode and a negatively charged cathode are suspended in sea water with an electric current flowing beetween them, calcium to the structure (cathode). The result is calcium cabonate Sub-marine flora and corals adhere to $\mathrm{CaCO} 3$ and grow quickly.
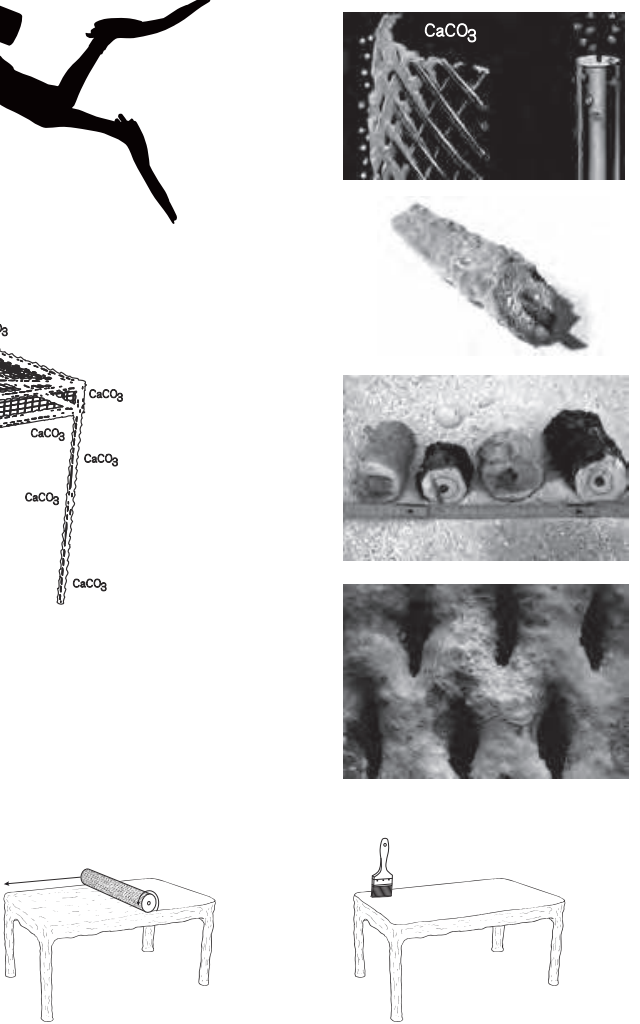

4_Rectifying the using surfaces
5_Fixing the material with bio-resin

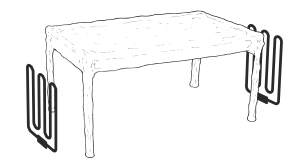

3_Drying the structure 




8 à 10. Tout comme les structures des récifs Biorock, le squelette de mobilier en accrétion minérale est construit en fers à béton assemblés et soudés sur la plage de l'île de Gili Trawangan en Indonésie. Ce squelette de mobilier est la base du premier prototype de mobilier M.A.F.
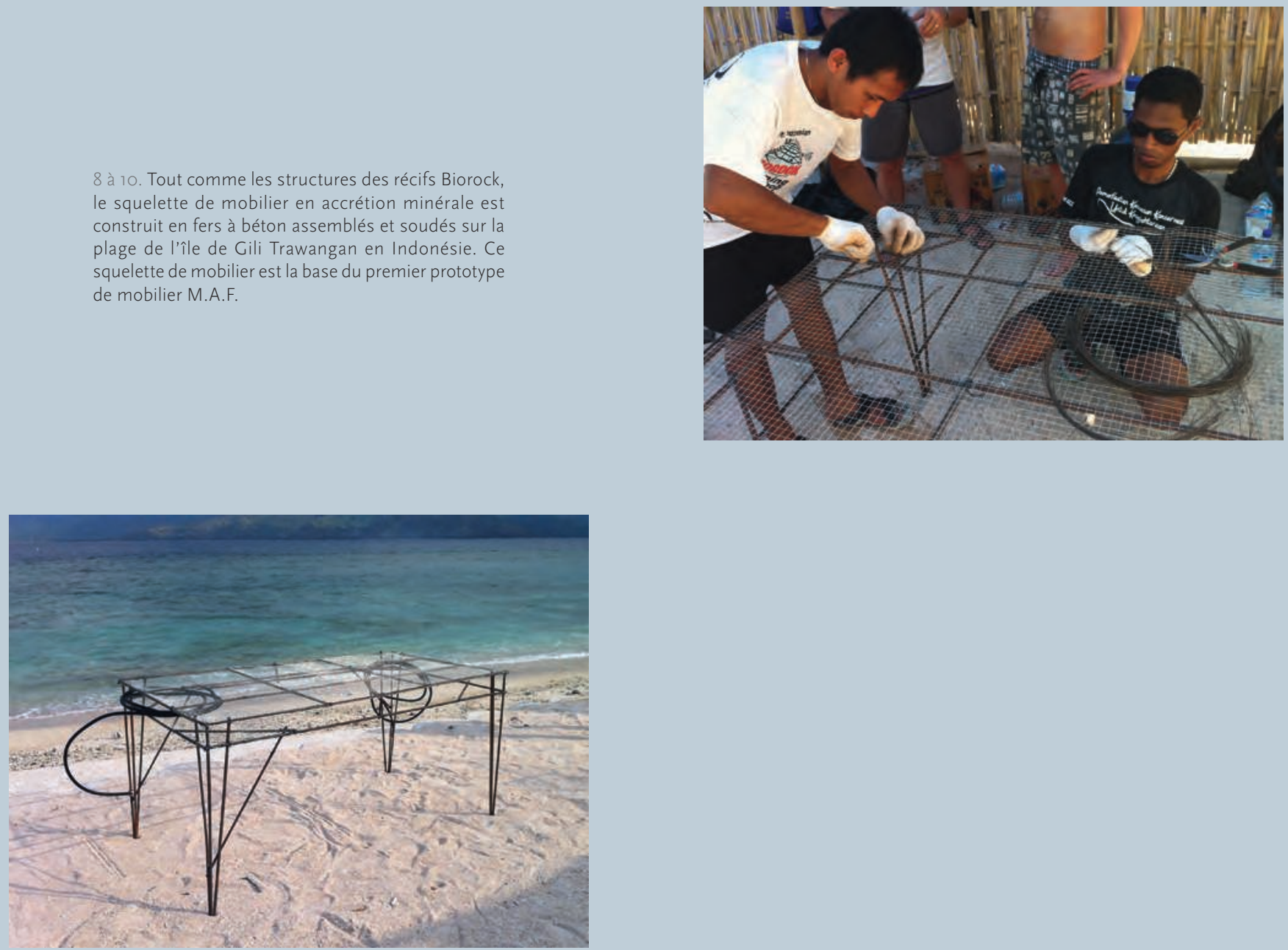



11 à 13. Le squelette de mobilier réalisé sur la plage est ensuite acheminé sur son lieu d'implantation par bateau. II est immergé et raccordé au courant électrique basse tension et fait ainsi office de cathode.

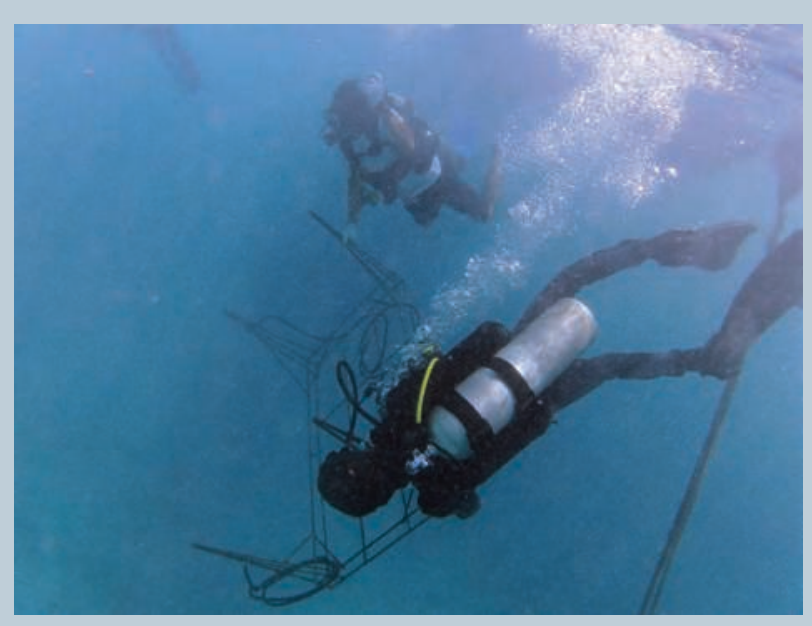



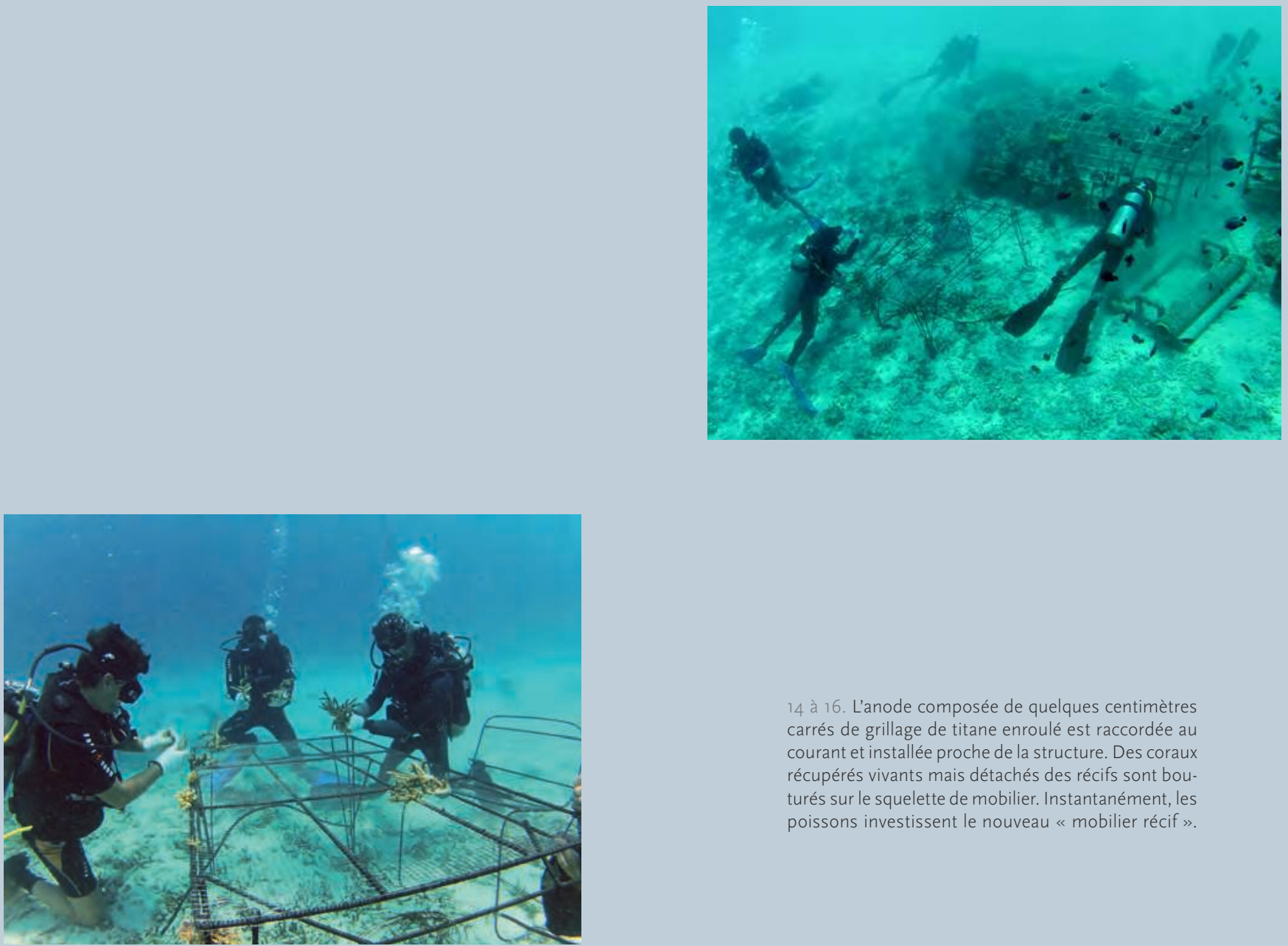

14 à 16. L'anode composée de quelques centimètres carrés de grillage de titane enroulé est raccordée au courant et installée proche de la structure. Des coraux récupérés vivants mais détachés des récifs sont bouturés sur le squelette de mobilier. Instantanément, les poissons investissent le nouveau « mobilier récif ».

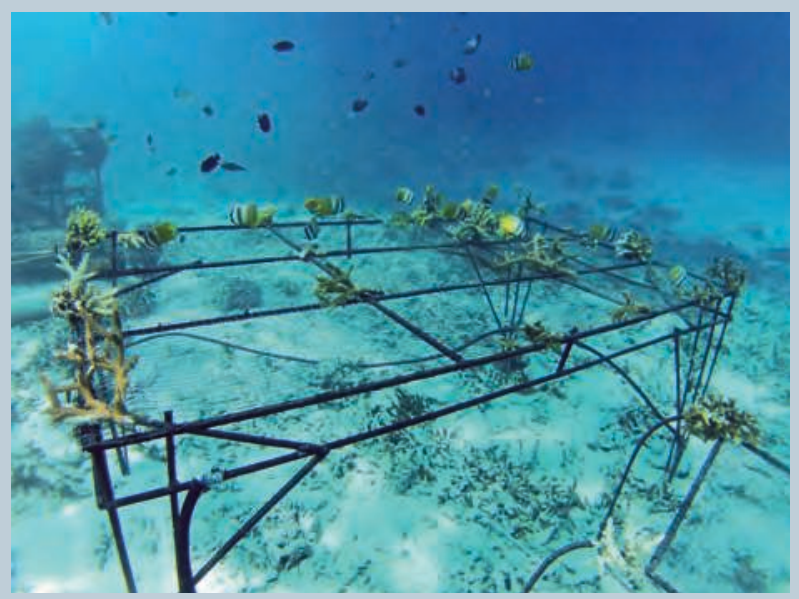



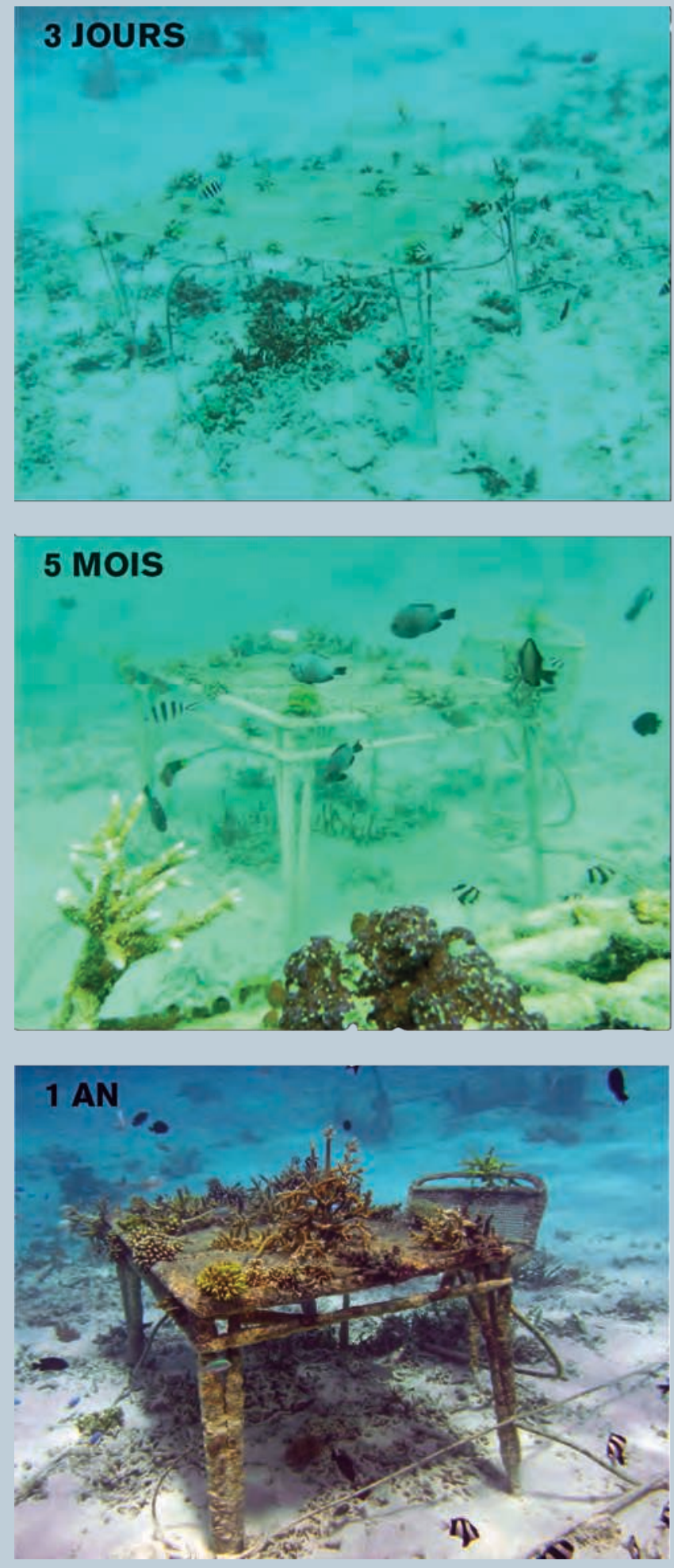




18 à 20. Un prototype de banc, une série d'objets tests ainsi qu'un abri à tortues ont été immergés en octobre 2016 dans les bassins de Kélonia à Saint-Leu sur l'île de la Réunion. C'est une nouvelle étape pour affiner les caractéristiques du dessin, le réglage du dispositif et valider l'intégralité de la chaîne de production. 


\section{Notes}

1. Pour exemple, le projet d'embellissement de la place Léon Aucoc à Bordeaux par le duo d'architectes Lacaton et Vassal. Les architectes ont en effet convenu qu'il était inapproprié d'engager un projet d'embellissement pour une place où « qualité, charme et vie existent » et en font une place « déjà belle ». Seuls des travaux d'entretien, simples et immédiats ont été engagés.

2. Article écrit pour l'ouvrage Les 101 mots du matériau dans le design sous la direction de Daniel Kula, Archibooks - Paris 2014.

3. Une donnée qui permet de mieux saisir les enjeux du débat concernant l'accord sur la tolérance au réchauffement entre $1,5^{\circ}$ et $2^{\circ}$ lors de la COP 21.

4. El Niño est à l'origine un courant océanique. Phénomène climatique singulier, il se traduit par une hausse anormale de la température à la surface de l'eau (10 mètres environ) dans la partie Est de l'océan Pacifique Sud. Il est synonyme d'inondations et de sècheresses records sur une grande partie du globe.

5. La grande barrière de corail - 2600 kilomètres est située au large de l'Australie, elle a été déclarée patrimoine mondiale de l'UNESCO en 1981.
6. Institut français de recherche pour l'exploitation de la mer.

7. La biogéochimie est la discipline scientifique qui traite de la transformation et du devenir de la matière, notamment de la matière organique et des éléments majeurs (C carbone, $\mathrm{N}$ azote, $\mathrm{P}$ phosphore, $\mathrm{S}$ soufre, etc.) dans la biosphère, par l'effet des processus biologiques, chimiques et géologiques. https://www.aquaportail.com/definition-4861-biogeochimie.html.

8. Ses recherches sur la question se trouvent rassemblées dans l'ouvrage Innovative Methods of Marine Ecosystem Restoration, Thomas J. Goreau \& Robert Kent Trench (dir.), CRC press, 2013.

9. Une partie de ses écrits et recherches sont disponibles sur le site internet www.wolfhilbertz.com.

10. Les posidonies sont de longues algues qui ressemblent à des tagliatelles vertes.

11. L'obtention de l'allocation de soutien pour le développement d'une recherche artistique du Centre National d'Arts Plastique obtenue en 2012, a permis la participation au workshop mené par Thomas J. Goreau et Delphine Robbe.

12. Autour de 1500 .

\section{L'auteur}

David Enon, designer indépendant, chemine entre projets de commandes, de recherche et enseignement. Présentes à la Tools Galerie (Paris) et à la Galerie MICA (Rennes), dans la collection du FNAC (Fonds national d'art contemporain) 2011, ses réalisations interrogent les modes de production des formes matérielles de notre environnement. Euvrant actuellement à la production de mobilier en récifs artificiels - projet pour lequel il a reçu une allocation de recherche du Cnap (Centre national des arts plastiques), il enseigne à l'école supérieure des beaux-arts Tours Angers Le Mans.

\section{Iconographie}

Image d'ouverture. Premier prototype de mobilier en accrétion minérale - M.A.F. Un an après sa mise à l'eau au large de l'île de Gili Trawangan en Indonésie. (C Foued Kaddachi, 2013.

$1 \& 2$. DR.

$3 \& 4$. Foued Kaddachi, 2013.
5 à 7 . Maquettes et poster du projet M.A.F. téléchargeable [http://david.enon.free.fr/vrac/Mineral\%20Accretion\%20Furniture/Poster\%20MAF.pdf], @ David Enon, 2011.

8 à 17. Phases de construction et de mise à l'eau (2012), et de croissance du projet M.A.F. (C) David Enon.

18 à 20. C) David Enon et Stéphane Ciccione, 2016.

\section{Pour citer l'article}

Enon, D. 2017 «Mineral Accretion Factory ou la mer comme usine nouvelle», TechniquesE Culture 67 « Low tech? Wild tech!», p. 240-257. 